Letter

\title{
Investigation on the Overshoot of Transient Open-Circuit Voltage in Methylammonium Lead Iodide Perovskite Solar Cells
}

\author{
Chunhai Li, Longfeng Lv, Liang Qin, Lijie Zhu, Feng Teng, Zhidong Lou, Zhenbo Deng, \\ Yufeng $\mathrm{Hu}$, Qiuhong Cui and Yanbing Hou *
}

Key Laboratory of Luminescence and Optical Information, Ministry of Education, Beijing JiaoTong University, Beijing 100044, China; 12118408@bjtu.edu.cn (C.L.); 11118405@bjtu.edu.cn (L.L.); 14118430@bjtu.edu.cn (L.Q.); 12118410@bjtu.edu.cn (L.Z.); fteng@bjtu.edu.cn (F.T.); zhdlou@bjtu.edu.cn (Z.L.); zbdeng@bjtu.edu.cn (Z.D.); yfhu@bjtu.edu.cn (Y.H.); qiuhcui@bjtu.edu.cn (Q.C.)

* Correspondence: ybhou@bjtu.edu.cn; Tel: +86-010-51684860

Received: 7 November 2018; Accepted: 26 November 2018; Published: 29 November 2018

\begin{abstract}
Although the performance of hybrid organic-inorganic perovskite solar cells (PSCs) is encouraging, the detailed working principles and mechanisms of PSCs remain to be further studied. In this work, an overshoot phenomenon of open-circuit voltage $\left(V_{o c}\right)$ was observed when the illumination light pulse was switched off. The evolution of the $V_{o c}$ overshoot was systematically investigated along with the intensity and the width of the light pulse, the background illumination, and pretreatment by different bias. Based on the experimental results, we could conclude that the $V_{o c}$ overshoot originated from carrier motion against carrier collection direction, which happened at the ionic-accumulation-induced band bending areas near the interfaces between the perovskite active layer and the two carrier transport layers. The investigation on the $V_{o c}$ overshoot can help us to better understand ionic migration, carrier accumulation, and recombination of PSCs under open-circuit conditions.
\end{abstract}

Keywords: overshoot; transient photovoltage; perovskite solar cell; ionic migration

\section{Introduction}

Hybrid organic-inorganic perovskite solar cells (PSCs) have attracted a lot of attention due to their rapidly increased power conversion efficiency (PCE) from 3.8\% [1] to 23.2\% [2]. However, the detailed working principles and mechanisms of PSCs are still not clear. The possible reasons for this phenomenon include ionic migration [3-6], ferroelectricity [7,8], charge trapping/detrapping [9,10], and capacitive effects [11].

Among these mechanisms, ionic migration has been considered to be the main reason for the hysteresis behavior in PSCs [12]. Therefore, much attention has been focused on the role of ions/vacancies driven by the built-in, photogenerated, and external electric fields [5,6]. To minimize hysteresis, some strategies have been proposed, such as preparing the perovskite crystal with larger grain size [11], using mesoporous titanium dioxide $\left(\mathrm{m}-\mathrm{TiO}_{2}\right)$ layers as the electron transport layer (ETL) [13], and using fullerene as the passivation layer to modify $\mathrm{TiO}_{2}$ [14] or the perovskite layer [15]. However, the method to avoid hysteresis with fullerene derivative as passivation layer does not seem to work all the time. In Khadka et al.'s work, PSCs with fullerene derivatives still showed hysteresis more or less [16]. Meanwhile, even some hysteresis-free PSCs still showed hysteresis behavior at low temperature [17]. Calado et al. found that ionic migration also existed in PSCs with minimal hysteresis [18]. 
Transient photovoltage (TPV), proposed as a tool to study the recombination of charge carriers in semiconductor diodes [19-21], has been widely used to investigate charge generation and recombination processes in solar cells [22-24]. In recent years, several groups have studied the dynamic characteristics and hysteresis of PSCs by TPV measurements. For example, Baumann et al. found that the recombination dynamics in all time regimes were dependent on the starting illumination intensity [25]. O'Regan et al. proved that hysteresis was not caused by the change of recombination rate and charge separation efficiency [26].

In this report, we performed TPV measurement on methylammonium lead iodide $\left(\mathrm{MAPbI}_{3}\right) \mathrm{PSCs}$ with rutile titanium dioxide $\left(\mathrm{R}-\mathrm{TiO}_{2}\right)$ nanocrystals as the ETL and observed an unpredictable overshoot of $V_{o c}$, instead of a descent as expected, when the illumination was switched off. This phenomenon was never observed in our previous work on organic solar cells (shown in Figure S1). O'Regan et al. had also observed a similar phenomenon, but they had attributed the overshoot to noise [26]. After investigating the evolution of the overshoot with various kinds of pulse light illumination, we suggest that the observed $V_{o c}$ overshoot resulted from ionic migration.

\section{Experimental Section}

\subsection{Materials}

Lead iodide $\left(\mathrm{PbI}_{2}\right)$, tert-butylpyridine (t-BP), and lithium-bis(trifluoromethanesulfonyl)imide (Li-TFSI) was purchased from Sigma-Aldrich, (Shanghai, China). Methylammonium iodide (MAI) was purchased from Dyesol, (Queanbeyan, Australia). 2,2',7,7'-tetrakis-(N,N-di-4-methoxyphenylamine)9,9'-spirobifluorene (spiro-OMeTAD) was purchased from 1-Material, (Dorval, Canada). All the other chemicals were purchased from Alfa Aesar (Shanghai, China) and were used as received.

\subsection{Preparation of $\mathrm{R}-\mathrm{TiO}_{2}$ Nanocrystals}

The ligand-free $\mathrm{R}-\mathrm{TiO}_{2}$ nanocrystals were made using an earlier reported method [27]. First, $2 \mathrm{~mL}$ titanium trichloride $\left(\mathrm{TiCl}_{3}, 15.0-20.0 \%\right.$ basis in $\left.30 \% \mathrm{HCl}\right)$ and $1 \mathrm{~mL}$ stannic chloride $\left(\mathrm{SnCl}_{4}\right)$ aqueous solution $(0.5 \mathrm{M})$ were added into $60 \mathrm{~mL}$ ethanol. Then, the mixed solution was placed in a drying oven at $75{ }^{\circ} \mathrm{C}$ for $8 \mathrm{~h}$ to get $\mathrm{R}-\mathrm{TiO}_{2}$ nanocrystals suspension. After being purified by ethanol and dispersed into water, a $12 \mathrm{mg} / \mathrm{mL} \mathrm{R}-\mathrm{TiO}_{2}$ nanocrystal aqueous solution was obtained.

\section{3. $\mathrm{MAPbI}_{3}$ PSC Fabrication}

The indium tin oxide (ITO)-coated glass substrates were cleaned by a neutral cleaning solution and then given an ultrasonic bath in deionized water, acetone, and isopropyl alcohol (IPA) for $20 \mathrm{~min}$ each. After $3 \mathrm{~min}$ ultraviolet-ozone treatment, $\mathrm{TiO}_{2}$ nanocrystal layer with the thickness of $\sim 30 \mathrm{~nm}$ was spin-coated on the ITO-coated glass substrates, followed by drying on a hotplate at $100{ }^{\circ} \mathrm{C}$ in the air and at $120^{\circ} \mathrm{C}$ in a nitrogen-filled glovebox for $15 \mathrm{~min}$ each. The device fabrication steps were all carried out in the glovebox. We used a previously reported two-step spin-coating procedure [28] to form a $\mathrm{MAPbI}_{3}$

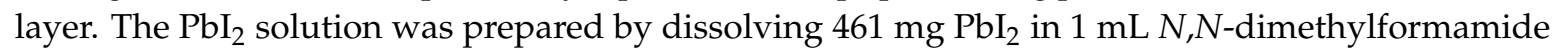
(DMF) under stirring at $70{ }^{\circ} \mathrm{C}$ overnight. The $\mathrm{PbI}_{2}$ solution was spin-coated on $\mathrm{TiO}_{2}$ film at $3000 \mathrm{rpm}$ for $15 \mathrm{~s}$ followed by drying at $70^{\circ} \mathrm{C}$ and cooling to room temperature. Next, $150 \mu \mathrm{L}$ of MAI solution (12 mg in 1mL IPA) was loaded on the $\mathrm{PbI}_{2}$-coated substrate and kept still for $40 \mathrm{~s}$, then spun at $9000 \mathrm{rpm}$ for $3 \mathrm{~s}$ and annealed at $90{ }^{\circ} \mathrm{C}$ for $30 \mathrm{~min}$. The spiro-OMeTAD solution was prepared by dissolving $68 \mathrm{mg}$ spiro-OMeTAD in $950 \mu \mathrm{L}$ chlorobenzene with $28 \mu \mathrm{L} t$-BP and $17.5 \mu \mathrm{L} \mathrm{Li}$-TFSI solution (520 mg Li-TFSI in $1 \mathrm{~mL}$ acetonitrile) as additives. This solution was spin-coated on the perovskite film as hole transport layer (HTL) at $5000 \mathrm{rpm}$ for $50 \mathrm{~s}$. A $\sim 10 \mathrm{~nm}$ molybdenum oxide $\left(\mathrm{MoO}_{3}\right)$ layer and a $\sim 200 \mathrm{~nm}$ Ag electrode were finally evaporated in a vacuum chamber with a pressure of $3 \times 10^{-5} \mathrm{~Pa}$, successively. The effective electrode area was $4.5 \mathrm{~mm}^{2}$, controlled by a mask. 


\section{Results and Discussion}

The PSC device structure was ITO/ $\mathrm{TiO}_{2} / \mathrm{MAPbI}_{3} /$ spiro-OMeTAD $/ \mathrm{MoO}_{3} / \mathrm{Ag}$. A cross-sectional image of the PSC taken by Hitachi (S-4800, Tokyo, Japan) field emission scanning electron microscope (SEM) is shown in Figure 1a. The surface SEM image of the perovskite layer is shown in Figure S2. The energy level diagram is shown in Figure $1 \mathrm{~b}$. The current density-voltage $(J-V)$ characteristics of PSCs were measured in the nitrogen atmosphere with a computer-controlled Keithley 6430 source measure unit under $100 \mathrm{~mW} / \mathrm{cm}^{2}$ illumination generated by the AM $1.5 \mathrm{G}$ solar simulator (XEC-301S, SAN-EI Electric, Osaka, Japan). The light intensity was calibrated by a silicon detector. We scanned the sample from 2 to $-0.5 \mathrm{~V}$ (reverse scan) and then scanned it in the opposite direction (forward scan) continuously. The $J-V$ characteristics of the device are shown in Figure $1 \mathrm{c}$ and Table S1. The incident photon-to-current conversion efficiency (IPCE) was measured by Zolix Solar Cell Scan 100, Beijing, China. The IPCE curve and the integrated short-circuit current density $\left(J_{s c}\right)$ of the PSC device are shown in Figure 1d.

(a)
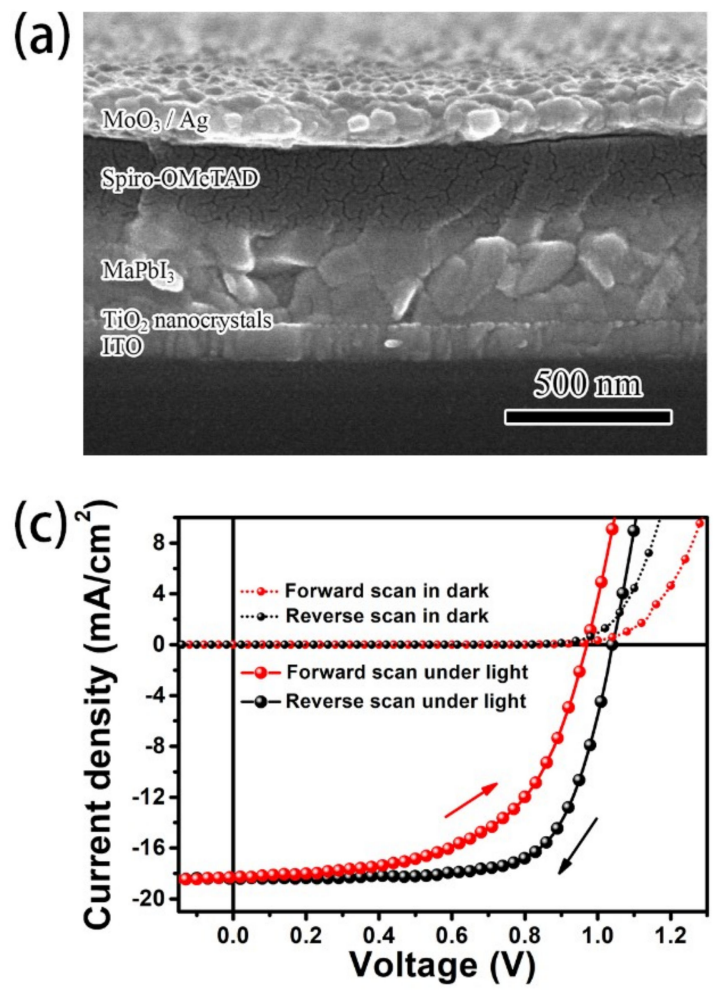
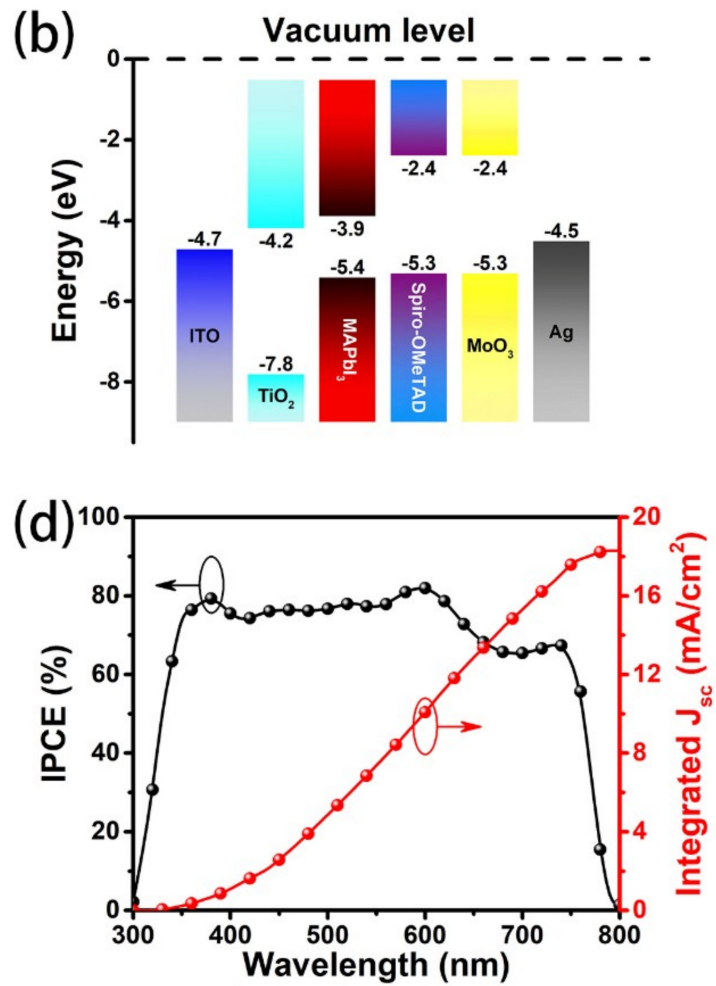

Figure 1. (a) Cross-sectional SEM image, (b) energy level diagram, (c) current density-voltage ( $J$ - $V$ ) characteristics, and (d) dependence of photon-to-current conversion efficiency (IPCE) on wavelength and the integrated short-circuit current density $\left(J_{s c}\right)$ of the perovskite solar cells (PSC) device.

For the TPV measurement, a pulse generator (WF1946B, NF company, Yokohama, Japan) was used to drive a white light-emitting diode (LED) to generate light pulses with different intensities. The samples were kept in a chamber filled with nitrogen $\left(\mathrm{N}_{2}\right)$ to avoid degradation. The setup for TPV measurement is given in Figure 2a. The TPV signal was taken by an amplifier (OPA1S2384, Texas Instruments, Dallas, TX, USA) with input impedance of $10^{13} \Omega$. For the TPV measurement under the presence of light bias, another white LED driven by a DC power supply (IT6133B, ITECH, New Taipei City, Taiwan) was used to provide a continuous background illumination as photobias. 
(a)

Pulse Generator

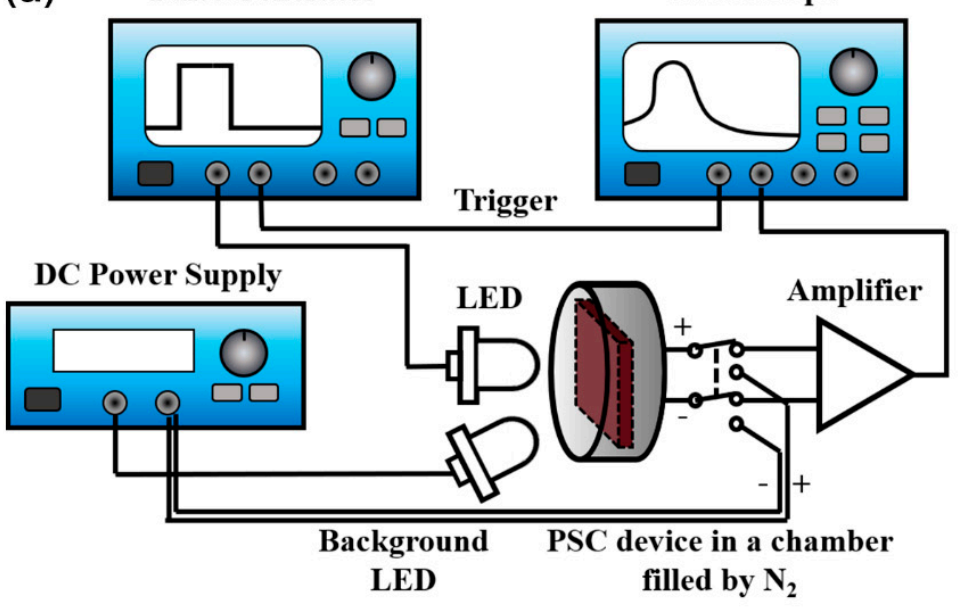

(b)
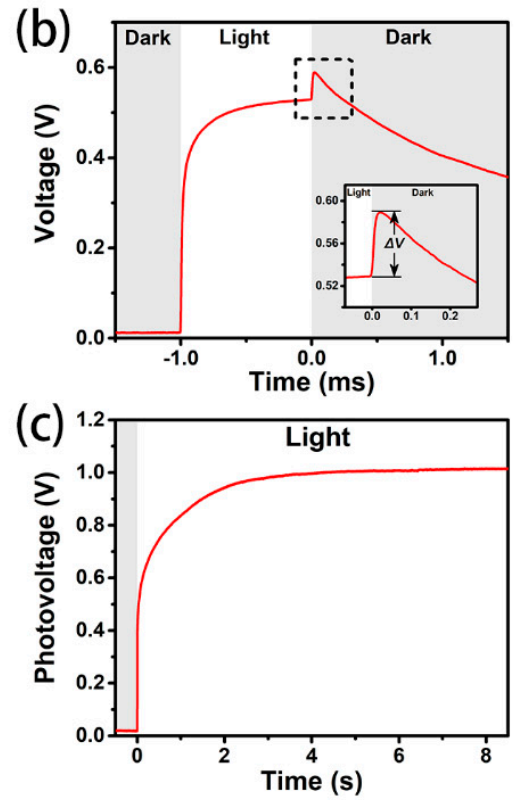

Figure 2. (a) Schematic diagram of transient photovoltage (TPV) measurement and $(\mathbf{b}, \mathbf{c})$ time-dependent photovoltage curve of TPV measurement on the PSC device under the illumination of 1-sun-equivalent pulse intensity when the light is (b) on at $-1 \mathrm{~ms}$ and off at $0 \mathrm{~ms}$ and (c) on at $0 \mathrm{~s}$ and off over $9 \mathrm{~s}$.

Figure $2 \mathrm{~b}$ shows a typical time-dependent voltage $(V-T)$ curve of TPV measurement on the PSC with the structure of $\mathrm{TiO}_{2} / \mathrm{MAPbI}_{3} /$ spiro-OMeTAD. The sample was illuminated by a pulsed white LED with 1-sun-equivalent intensity. The width and repetition rate of the light pulse were $1 \mathrm{~ms}$ and $1 \mathrm{~Hz}$, respectively. The gray area and white area in Figure $2 \mathrm{~b}$ indicate light being off and on, respectively. As shown in this figure, the whole $V$ - $T$ curve includes four parts: a fast-rising period ( $\sim 0.1 \mathrm{~ms})$ at the very beginning of the illumination, a slow-rising period in the remaining time of illumination, a fast upshift (overshoot, $\Delta V$ ) after light pulse off, and a continuous descent after the upshift. Herein, our work is focused on investigating the evolution of the $V_{o c}$ overshoot under different illumination and device pretreatments.

Figure 3a shows the transient $V$ - $T$ curves of the PSC device under the illumination light pulses with different intensities. When the intensity of illumination light pulse increased from 82 to $103 \mathrm{~mW} / \mathrm{cm}^{2}$, the upshift of $V_{o c}$ increased from 0 to $75 \mathrm{mV}$. Figure $3 \mathrm{~b}$ shows the transient $V$ - $T$ curves of the PSC under illumination with different pulse widths. The illumination pulse started at $-400,-100,-10$, and $-1 \mathrm{~ms}$. The upshift of $V_{o c}$ decreased from 67.7 to $8.4 \mathrm{mV}$ along with the increase in the pulse width from 1 to $400 \mathrm{~ms}$. Figure 3c presents the transient $V$ - $T$ curves of the PSC device under the presence of a light bias with different intensities. Here, the pink areas indicate that the pulse illumination is off and the light bias is on. It can be observed that when the light intensity increased from 0 to $85 \mathrm{~mW} / \mathrm{cm}^{2}$, the overshoot decreased from 59.1 to $1.2 \mathrm{mV}$ and nearly disappeared. Figure $3 \mathrm{~d}$ shows the transient $V$ - $T$ curve of the PSC device under the illumination of double light pulses with an $80 \mu$ interval. The $V_{o c}$ showed a sudden descent of $58.0 \mathrm{mV}$ when the second light pulse was on. The decent of $V_{o c}$ equaled the overshoot of $58.9 \mathrm{mV}$ when the first light pulse passed through. All the results presented in Figure 3 prove the existence of $V_{o c}$ overshoot in PSCs with $\mathrm{TiO}_{2}$ as ETL. 

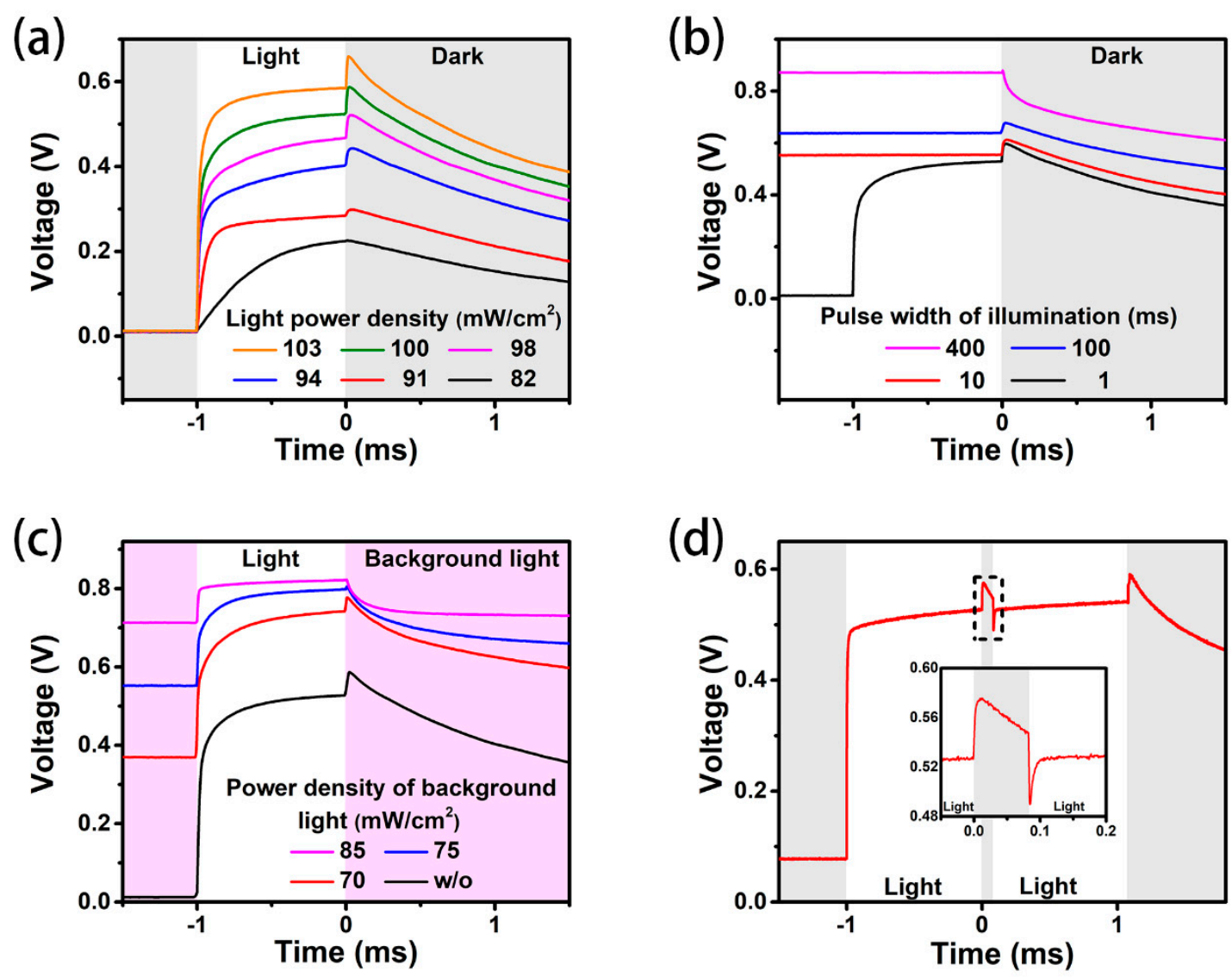

Figure 3. TPV measurement of PSC devices: (a) the dependence of TPV on the intensity of light pulses; (b) the dependence of TPV on the width of light pulses; (c) the TPV obtained under the presence of light bias with different intensities; (d) the TPV obtained under the illumination of double light pulses with an $80 \mu$ s interval. All curves, except for (a), were obtained under the illumination of 1-sun-equivalent pulse intensity. Except (b), the light is on at $-1 \mathrm{~ms}$ and off at $0 \mathrm{~ms}$.

In general, $V_{o c}$ originates from the splitting of electron and hole quasi-Fermi energy levels actuated by light illumination:

$$
V_{o c}=\frac{1}{q}\left(E_{F N}-E_{F P}\right)
$$

where $q$ is the elementary charge, and $E_{F N}$ and $E_{F P}$ are the electron and hole quasi-Fermi levels, respectively [29]. In TPV measurement, $V_{o c}$ can be related to the charge density in the device, assuming that the photovoltage is equal to the quasi-Fermi level splitting:

$$
V_{o c}=\frac{k_{B} T}{q} \ln \left(\frac{n p}{n_{0} p_{0}}\right)
$$

where $k_{B}$ is the Boltzmann constant; $T$ is the temperature; $q$ is the elementary charge; $n$ and $p$ are the total concentrations of electrons and holes; and $n_{0}$ and $p_{0}$ are the intrinsic electron and hole concentrations, respectively [25]. For a device kept under open-circuit condition, no photogenerated charge carriers are produced when the illumination is switched off, and the number of existing electrons and holes keeps decreasing due to the recombination within the device, resulting in a continuous descent of $V_{o c}$ theoretically. However, the TPV curve of PSC we obtained showed an overshoot of voltage when the illumination was off, which was in contradiction with the above analysis for normal solar cells.

Indeed, under the illumination of the millisecond-timescale light pulse, the photovoltage was different from the $V_{o c}$ obtained from $J-V$ measurement under continuous illumination with the same light intensity. Our time-dependent photovoltage measurements revealed that it took more than $8 \mathrm{~s}$ 
for the photovoltage to reach its maximum $V_{o c}$ under a continuous illumination of 1-sun-equivalent intensity (Figure 2c). According to the literature, the slow rise of $V_{o c}$ is associated with ionic migration under illumination [30]. Normally, $V_{o c}$ is related to the number and the distribution of charge carriers. Nie et al. demonstrated that photogenerated carriers could populate the light-activated trap states under light and relax in dark using photocurrent transient measurements and PL spectra [31].

Previous researches have reported that ions do exist and are able to migrate in the $\mathrm{MAPbI}_{3}$ perovskite layer $[4,18]$. Under the drive of the built-in electric field, anions migrate and accumulate at the interface between $\mathrm{TiO}_{2}$ / perovskite, which would lead to the upward bending of energy band and the formation of barrier for electrons. The same thing also happened at the interface between spiro-OMeTAD/perovskite for holes. The energy level diagrams of PSC without and with ionic accumulation are given in Figure 4. For the PSC without ionic accumulation, all the photogenerated electrons/holes drifted to cathode/anode under the modification of the built-in electric field directing from cathode to anode, except some carriers that recombined via carrier-carrier recombination and defect recombination. When the PSC was applied at a negative bias or a bias less than the built-in potential, the mobile anions were driven to the region close to $\mathrm{TiO}_{2}$ electron collection layer, and the cations migrated to spiro-OMeTAD hole collection layer, which led to the energy band bending in both regions (as shown in Figure $4 \mathrm{~b}$ ). Due to the energy band bending, the photogenerated carriers in these regions were driven to the wrong directions with respect to charge collection, leading to an increased accumulation of minority carriers at two perovskite interfaces. This meant that the direction of the electric field in these two regions was opposite to that of the built-in electric field. The inverted electric field reduced the collection efficiency and changed the distribution of the charge carrier, which caused an obvious decrease in $V_{o c}$. The intensity of the opposite electric field increased with the number of carriers generated close to the carrier transport layers. Because the light goes through the PSC from the $\mathrm{TiO}_{2}$ side, the interface between $\mathrm{TiO}_{2}$ and perovskite should contribute more to the inverted electric field. When the light was turned off, no carriers were generated, and inverted electric field disappeared, which led to an enhanced $V_{o c}$. Although the number of carriers decreased because of recombination, the redistribution of carriers was much faster than the recombination of carriers. The rising time for the overshoot of $V_{o c}$ was about $20 \mu \mathrm{s}$, which implied that the overshoot was an electronic behavior, instead of an ionic behavior. If PSC was applied at a positive bias larger than the built-in potential, the anions and cations drifted toward to spiro-OMeTAD and $\mathrm{TiO}_{2}$, respectively. They accumulated close to the respective interfaces, and the energy bands in the cation and anion accumulation region bent downward and upward, respectively, as shown in Figure 4c. In this situation, the direction of carrier-motion-induced electric field was the same as that of the built-in electric field in the energy band bending regions, which benefited to the carrier collection and resulted in an increase of $V_{o c}$. The difference in ionic accumulation is the main reason for hysteresis in PSCs [4].

The overshoot enhanced with the increasing intensity of light pulse, as shown in Figure 3a, because the intense light pulse generated more carriers, which moved to opposite directions, thus enhancing the $V_{o c}$ overshoot. When the width of the light pulse was increased, more carriers were generated, thus the $V_{o c}$ became larger. By contrast, the overshoot abated with the increasing width of the light pulse. When the width of the light pulse was $400 \mathrm{~ms}$, the overshoot of $V_{o c}$ nearly disappeared. With increasing width of the light pulse, more electrons and holes were generated, and the energy valley near the interface of $\mathrm{TiO}_{2}$ / perovskite was gradually filled with more electrons. Some of them would have got over the barrier caused by ionic accumulation, and the $V_{o c}$ overshoot became unobvious. The $V$-T curve measured under the illumination of a double light pulse further verified the existence of the reverted electric field. After the first light pulse passed through, the carriers generated by the first light pulse still stayed at conductive band or valence band for hundreds of milliseconds. When the second light pulse was on, the carrier motion against the collection layers turned on the inverted electric field, thus causing a decrease in $V_{o c}$. 

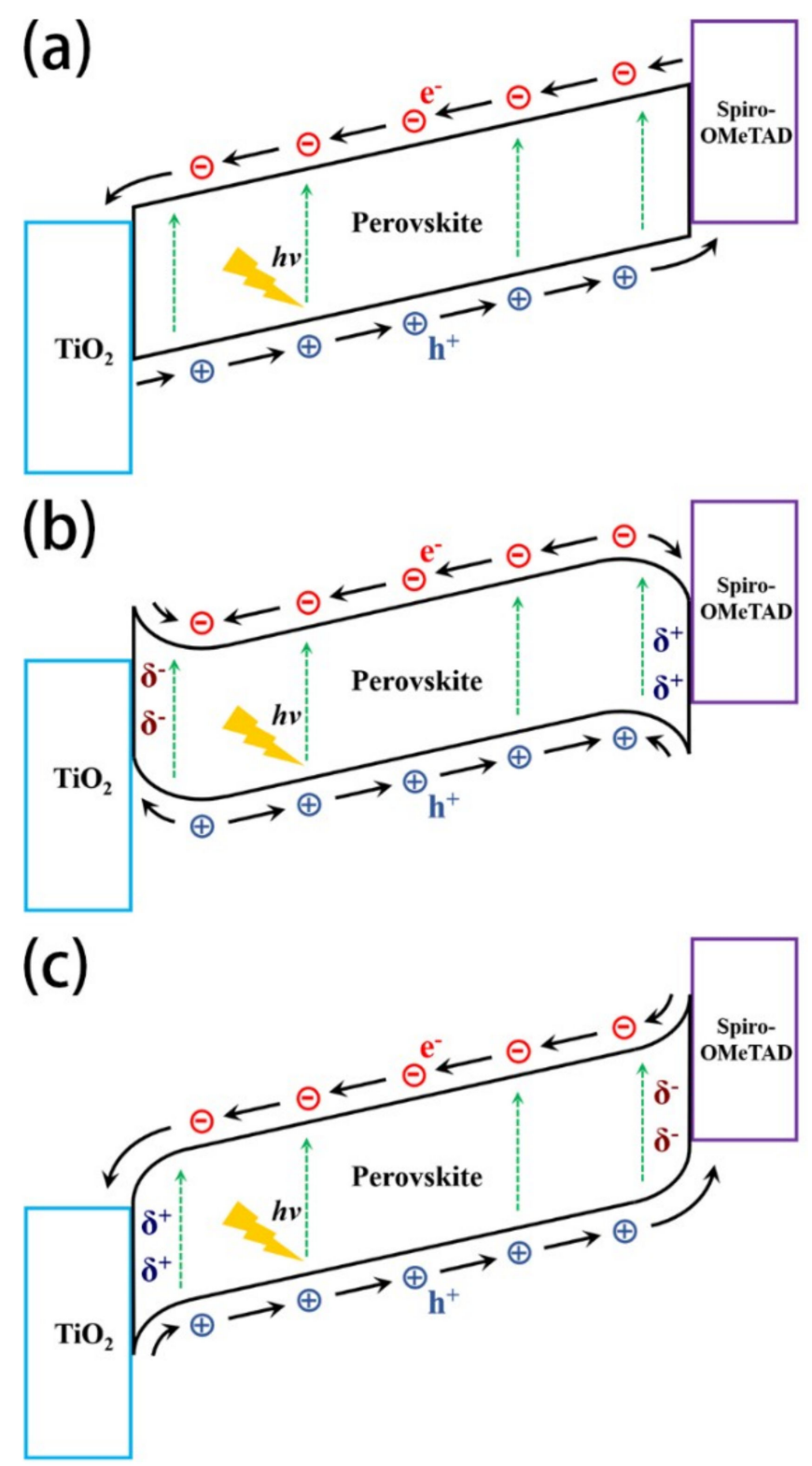

Figure 4. Schematic diagram of PSC device (a) without ionic accumulation; (b) with anions and cations accumulating at the interfaces of $\mathrm{TiO}_{2}$ /perovskite and spiro-OMeTAD/perovskite, respectively; (c) with cations and anions accumulating at the interfaces of $\mathrm{TiO}_{2}$ /perovskite and spiro-OMeTAD/perovskite, respectively.

As previously mentioned, when the PSC is applied at a positive bias higher than the built-in potential, the energy band will bend, as shown in Figure $4 \mathrm{c}$. In this situation, $V_{o c}$ overshoot should disappear. In order to verify this assumption, the PSCs were pretreated with different positive biases for $90 \mathrm{~s}$ before the TPV measurement. When the pretreatment bias increased from $0.9 \mathrm{~V}$ to $2.3 \mathrm{~V}$, the overshoot decreased from $59.1 \mathrm{mV}$ to $6.2 \mathrm{mV}$, as shown in Figure 5. This was direct evidence that the ionic formation induced by the built-in electric field was the origin of the $V_{o c}$ overshoot.

Weber et al. showed that the formation of ionic charges at the interfaces is the dominating factor for $J-V$ hysteresis [32]. Pockett et al. performed a similar TPV measurement at low temperature and attributed the $J-V$ hysteresis to ionic redistribution and interfacial recombination [33]. In this study, we changed the starting conditions of the ionic formation and observed the corresponding changes in the $V_{o c}$ overshoot. These results showed that the $V_{o c}$ overshoot was related to the starting condition of ionic formation. It is believed that the $V_{o c}$ overshoot should be associated with hysteresis. 


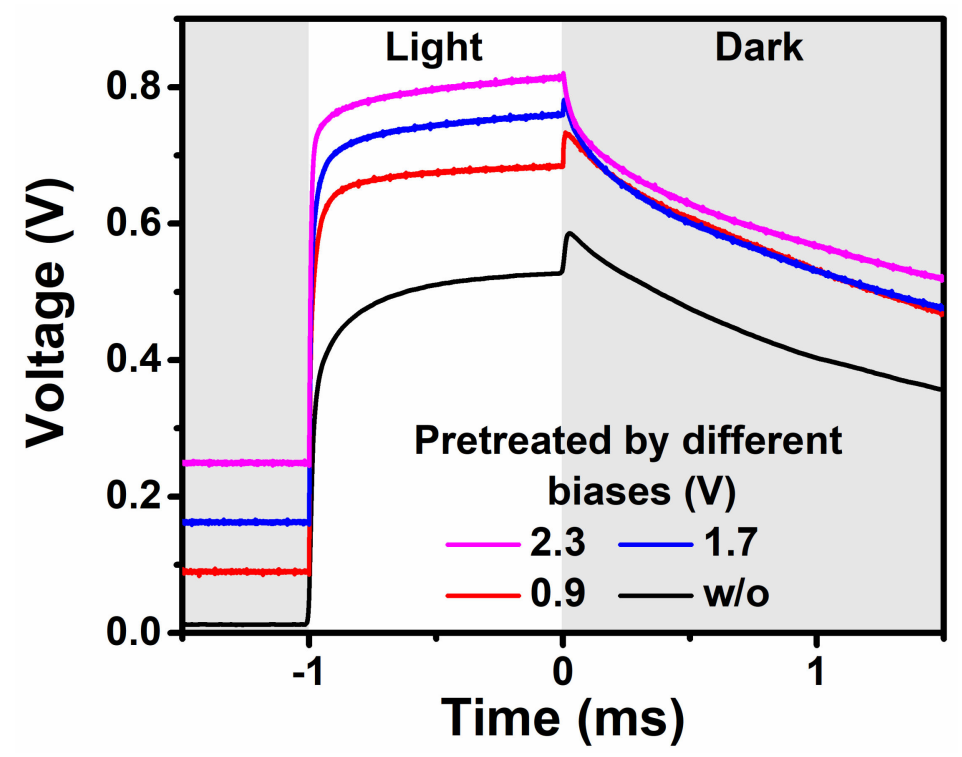

Figure 5. TPV of PSC pretreated with different electric biases for $90 \mathrm{~s}$ before TPV measurement.

\section{Conclusions}

TPV measurement is an effective technique to investigate PSCs. In this work, we performed this measurement on PSCs, and a $V_{o c}$ overshoot behavior was observed. The influence of several factors on the $V_{o c}$ overshoot was investigated, including the pulse illumination density, the pulse width of illumination, the background illumination, and the pretreatment of external electric field. The $V_{o c}$ overshoot mainly originated from the barrier-induced band bending that originated from ionic accumulation. This work provides a framework to understand the ionic-migration-induced phenomena of PSCs.

Supplementary Materials: The following are available online at http:/ / www.mdpi.com/1996-1944/11/12/2407/ s1, Figure S1: Transient $V$-T curve of organic solar cell with the structure of ITO/ $\mathrm{TiO}_{2} / \mathrm{PTB}_{\mathrm{P}} \mathrm{PC} \mathrm{C}_{71} \mathrm{BM} / \mathrm{MoO} / \mathrm{Ag}$ under the illumination of 1-sun-equivalent pulse intensity. The light is on at $-1 \mathrm{~ms}$ and off at $0 \mathrm{~ms}$. Figure S2. SEM image of the surface of perovskite layer. Table S1: Characteristics of PSC device.

Author Contributions: Conceptualization, C.L., L.L. and Y.H. (Yanbing Hou); Methodology, C.L., L.L. and Y.H. (Yanbing Hou); Software, Q.L.; Validation, F.T., Z.L., Y.H. (Yufeng Hu) and Z.D.; Formal Analysis, Y.H. (Yanbing Hou) and C.L.; Investigation, C.L.; Resources, L.Z.; Data Curation, C.L.; Writing-Original Draft Preparation, C.L.; Writing-Review \& Editing, Y.H. (Yanbing Hou), Z.L., L.Z. and Q.C.; Visualization, C.L.; Supervision, Y.H. (Yanbing Hou); Project Administration, Y.H. (Yanbing Hou) and F.T.

Funding: This work was supported by the National Key Research and Development Program of China (2016YFB0700703), the Natural Science Foundation of China (No. 61475017, 51603010, 61377028, 61475014, $61674012,61675018,61735004,61775011)$, and the Fundamental Research Funds for the Central Universities (2018JBM068).

Conflicts of Interest: The authors declare no conflicts of interest.

\section{References}

1. Kojima, A.; Teshima, K.; Shirai, Y.; Miyasaka, T. Organometal halide perovskites as visible-light sensitizers for photovoltaic cells. J. Am. Chem. Soc. 2009, 131, 6050-6051. [CrossRef] [PubMed]

2. Jeon, N.J.; Na, H.; Jung, E.H.; Yang, T.-Y.; Lee, Y.G.; Kim, G.; Shin, H.-W.; Seok, S.I.; Lee, J.; Seo, J. A fluorene-terminated hole-transporting material for highly efficient and stable perovskite solar cells. Nat. Energy 2018, 3, 682-689. [CrossRef]

3. Eames, C.; Frost, J.M.; Barnes, P.R.; O’Regan, B.C.; Walsh, A.; Islam, M.S. Ionic transport in hybrid lead iodide perovskite solar cells. Nat. Commun. 2015, 6, 7497. [CrossRef] [PubMed] 
4. Li, C.; Tscheuschner, S.; Paulus, F.; Hopkinson, P.E.; Kießling, J.; Köhler, A.; Vaynzof, Y.; Huettner, S. Iodine Migration and its Effect on Hysteresis in Perovskite Solar Cells. Adv. Mater. 2016, 28, 2446-2454. [CrossRef] [PubMed]

5. Seol, D.; Jeong, A.; Han, M.H.; Seo, S.; Yoo, T.S.; Choi, W.S.; Jung, H.S.; Shin, H.; Kim, Y. Origin of hysteresis in $\mathrm{CH}_{3} \mathrm{NH}_{3} \mathrm{PbI}_{3}$ perovskite thin films. Adv. Funct. Mater. 2017, 27, 1701924. [CrossRef]

6. O'Kane, S.E.J.; Richardson, G.; Pockett, A.; Niemann, R.G.; Cave, J.M.; Sakai, N.; Eperon, G.E.; Snaith, H.J.; Foster, J.M.; Cameron, P.J. Measurement and modelling of dark current decay transients in perovskite solar cells. J. Mater. Chem. C 2017, 5, 452-462. [CrossRef]

7. Frost, J.; Butler, K.T.; Brivio, F.; Hendon, C.H.; Schilfgaarde, M.V.; Walsh, A. Atomistic origins of high-performance in hybrid halide perovskite solar cells. Nano Lett. 2014, 14, 2584-2590. [CrossRef] [PubMed]

8. Frost, J.M.; Butler, K.T.; Walsh, A. Molecular ferroelectric contributions to anomalous hysteresis in hybrid perovskite solar cells. APL Mater. 2014, 2, 081506. [CrossRef]

9. Heo, J.H.; Song, D.H.; Han, H.J.; Kim, S.Y.; Kim, J.H.; Kim, D.; Shin, H.W.; Ahn, T.K.; Wolf, C.; Lee, T.W. Planar $\mathrm{CH}_{3} \mathrm{NH}_{3} \mathrm{PbI}_{3}$ perovskite solar cells with constant $17.2 \%$ average power conversion efficiency irrespective of the scan rate. Adv. Mater. 2015, 27, 3424-3430. [CrossRef] [PubMed]

10. Xu, J.; Buin, A.; Ip, A.H.; Li, W.; Voznyy, O.; Comin, R.; Yuan, M.; Jeon, S.; Ning, Z.; McDowell, J.J. Perovskite-fullerene hybrid materials suppress hysteresis in planar diodes. Nat. Commun. 2015, 6, 7081. [CrossRef] [PubMed]

11. Kim, H.S.; Park, N.G. Parameters Affecting I-V hysteresis of $\mathrm{CH}_{3} \mathrm{NH}_{3} \mathrm{PbI}_{3}$ perovskite solar cells: Effects of perovskite crystal size and mesoporous $\mathrm{TiO}_{2}$ layer. J. Phys. Chem. Lett. 2014, 5, 2927-2934. [CrossRef] [PubMed]

12. Elumalai, N.K.; Uddin, A. Hysteresis in organic-inorganic hybrid perovskite solar cells. Sol. Energy Mater. Sol. Cells 2016, 157, 476-509. [CrossRef]

13. Unger, E.L.; Hoke, E.T.; Bailie, C.D.; Nguyen, W.H.; Bowring, A.R.; Heumüller, T.; Christoforo, M.G.; Mcgehee, M.D. Hysteresis and transient behavior in current-voltage measurements of hybrid-perovskite absorber solar cells. Energy Environ. Sci. 2014, 7, 3690-3698. [CrossRef]

14. Wojciechowski, K.; Stranks, S.D.; Abate, A.; Sadoughi, G.; Sadhanala, A.; Kopidakis, N.; Rumbles, G.; Li, C.Z.; Friend, R.H.; Jen, A.K. Heterojunction modification for highly efficient organic-inorganic perovskite solar cells. ACS Nano 2014, 8, 12701-12709. [CrossRef] [PubMed]

15. Shao, Y.; Xiao, Z.; Bi, C.; Yuan, Y.; Huang, J. Origin and elimination of photocurrent hysteresis by fullerene passivation in $\mathrm{CH}_{3} \mathrm{NH}_{3} \mathrm{PbI}_{3}$ planar heterojunction solar cells. Nat. Commun. 2014, 5, 5784. [CrossRef] [PubMed]

16. Khadka, D.B.; Shirai, Y.; Yanagida, M.; Noda, T.; Miyano, K. Tailoring the open-circuit voltage deficit of wide-band-gap perovskite solar cells using alkyl chain-substituted fullerene derivatives. ACS Appl. Mater. Interfaces 2018, 10, 22074-22082. [CrossRef] [PubMed]

17. Bryant, D.; Wheeler, S.; O’Regan, B.C.; Watson, T.; Barnes, P.R.F.; Worsley, D.; Durrant, J. Observable hysteresis at low temperature in "hysteresis free" organic-inorganic lead halide perovskite solar cells. J. Phys. Chem. Lett. 2015, 6, 3190-3194. [CrossRef]

18. Calado, P.; Telford, A.M.; Bryant, D.; Li, X.; Nelson, J.; O’Regan, B.C.; Barnes, P.R.F. Evidence for ion migration in hybrid perovskite solar cells with minimal hysteresis. Nat. Commun. 2016, 7, 13831. [CrossRef] [PubMed]

19. Jain, S.C. Theory of photo induced open circuit voltage decay in a solar cell. Solid.State Electron. 1981, 24, 179-183. [CrossRef]

20. Green, M.A. Minority carrier lifetimes using compensated differental open circuit voltage decay. Solid State Electron. 1983, 26, 1117-1122. [CrossRef]

21. Green, M.A. Solar cell minority carrier lifetime using open-circuit voltage decay. Solar Cells 1984, 11, 147-161. [CrossRef]

22. Maurano, A.; Hamilton, R.; Shuttle, C.G.; Ballantyne, A.M.; Nelson, J.; O’Regan, B.; Zhang, W.; Mcculloch, I.; Azimi, H.; Morana, M. Recombination dynamics as a key determinant of open circuit voltage in organic bulk heterojunction solar cells: A comparison of four different donor polymers. Adv. Mater. 2010, 22, 4987-4992. [CrossRef] [PubMed]

23. Sudheendra Rao, K.; Mohapatra, Y.N. Open circuit voltage decay transients and recombination in bulk-heterojunction solar cells. Appl. Phys. Lett. 2014, 104, 203303. [CrossRef] 
24. Wang, H.Y.; Wang, Y.; Yu, M.; Han, J.; Guo, Z.X.; Ai, X.C.; Zhang, J.P.; Qin, Y. Mechanism of biphasic charge recombination and accumulation in $\mathrm{TiO}_{2}$ mesoporous structured perovskite solar cells. Phys. Chem. Chem. Phys. 2016, 18, 12128-12134. [CrossRef] [PubMed]

25. Baumann, A.; Tvingstedt, K.; Heiber, M.C.; Väth, S.; Momblona, C.; Bolink, H.J.; Dyakonov, V. Persistent photovoltage in methylammonium lead iodide perovskite solar cells. APL Mater. 2014, 2, 081501. [CrossRef]

26. O’Regan, B.C.; Barnes, P.R.; Li, X.; Law, C.; Palomares, E.; Marin-Beloqui, J.M. Optoelectronic studies of methylammonium lead iodide perovskite solar cells with mesoporous $\mathrm{TiO}_{2}$ : Separation of electronic and chemical charge storage, understanding two recombination lifetimes, and the evolution of band offsets during J-V hysteresis. J. Am. Chem. Soc. 2015, 137, 5087-5099. [CrossRef] [PubMed]

27. Zhu, L.; Lu, Q.; Lv, L.; Wang, Y.; Hu, Y.; Deng, Z.; Lou, Z.; Hou, Y.; Teng, F. Ligand-free rutile and anatase $\mathrm{TiO}_{2}$ nanocrystals as electron extraction layers for high performance inverted polymer solar cells. RSC Adv. 2017, 7, 20084-20092. [CrossRef]

28. Im, J.H.; Jang, I.H.; Pellet, N.; Grätzel, M.; Park, N.G. Growth of $\mathrm{CH}_{3} \mathrm{NH}_{3} \mathrm{PbI}_{3}$ cuboids with controlled size for high-efficiency perovskite solar cells. Nat. Nanotechnol. 2014, 9, 927-932. [CrossRef] [PubMed]

29. Elumalai, N.K.; Uddin, A. Open circuit voltage of organic solar cells: An in-depth review. Energy Environ. Sci. 2016, 9, 391-410. [CrossRef]

30. Pockett, A.; Eperon, G.E.; Sakai, N.; Snaith, H.J.; Peter, L.M.; Cameron, P.J. Microseconds, milliseconds and seconds: Deconvoluting the dynamic behaviour of planar perovskite solar cells. Phys. Chem. Chem. Phys. 2016, 19, 5959-5970. [CrossRef] [PubMed]

31. Nie, W.; Jean-Christophe, B.; Neukirch, A.J.; Kannatassen, A.; Hsinhan, T.; Manish, C.; Alam, M.A.; Sfeir, M.Y.; Claudine, K.; Jacky, E. Light-activated photocurrent degradation and self-healing in perovskite solar cells. Nat. Commun. 2016, 7, 11574. [CrossRef] [PubMed]

32. Weber, S.A.L.; Hermes, I.M.; Turren-Cruz, S.-H.; Gort, C.; Bergmann, V.W.; Gilson, L.; Hagfeldt, A.; Graetzel, M.; Tress, W.; Berger, R. How the formation of interfacial charge causes hysteresis in perovskite solar cells. Energy Environ. Sci. 2018, 11, 2404-2413. [CrossRef]

33. Pockett, A.; Carnie, M. Ionic influences on recombination in perovskite solar cells. ACS Energy Lett. 2017, 2, 1683-1689. [CrossRef] 